\title{
DETECTION OF SURFACE BRIGHTNESS FLUCTUATIONS IN NGC 4373 USING THE HUBBLE SPACE TELESCOPE ${ }^{1}$
}

Michael A. Pahre, ${ }^{2,3,4}$ Jeremy R. Mould, ${ }^{5}$ Alan Dressler,${ }^{6}$ Jon A. Holtzman, ${ }^{7}$ Alan M. Watson, ${ }^{8}$ John S. Gallagher III, ${ }^{8}$ Gilda E. Ballester, ${ }^{9}$ Christopher J. Burrows, ${ }^{10}$ Stefano Casertano, ${ }^{11}$ John T. Clarke, ${ }^{9}$ David Crisp, ${ }^{12}$ Richard E. Griffiths, ${ }^{11}$ Carl J. Grillmair, ${ }^{13}$ J. JefF Hester, ${ }^{14}$ John G. Hoessel,${ }^{8}$ Paul A. Scowen, ${ }^{14}$ Karl R. Stapelfeldt, ${ }^{13}$ John T. Trauger, ${ }^{13}$ and James A. WestPhal ${ }^{15}$

Received 1997 November 25 ; accepted 1998 November 12

\begin{abstract}
Surface brightness fluctuations (SBF) have been detected for three elliptical galaxies-NGC 3379 in the Leo group, NGC 4406 in the Virgo cluster, and NGC 4373 in the Hydra-Centaurus superclusterusing marginally sampled, deep images taken with the Planetary Camera of the WFPC2 instrument on the Hubble Space Telescope (HST). The power spectrum of the fluctuations image is well fitted by an empirical model of the point-spread function constructed using point sources identified in the field. Comparison with high-quality ground-based observations of all three galaxies show excellent agreement in the measurement of the distance modulus over a substantial range in distance. This demonstrates the capability of the Planetary Camera of WFPC2 to measure distances using the SBF technique despite the marginal sampling and small spatial coverage of the images. The residual variance due to unresolved sources in all three galaxies is only $2 \%-5 \%$ of the detected fluctuations signal, which confirms the advantage of HST imaging in minimizing the uncertainty of this SBF correction. Extensive consistency checks, including an independent SBF analysis using an alternate software package, suggest that our internal uncertainties are $<0.02 \mathrm{mag}$. The fluctuations magnitude for NGC 4373 is $I_{\mathrm{F} 814 \mathrm{~W}}=31.31 \pm$ $0.05 \mathrm{mag}$, corresponding to a distance modulus of $(m-M)_{0}=32.99 \pm 0.11$. This implies a peculiar velocity for this galaxy of $415 \pm 330 \mathrm{~km} \mathrm{~s}^{-1}$, which is smaller than derived from the $D_{n}-\sigma$ relation.

Subject headings: galaxies: distances and redshifts - galaxies: elliptical and lenticular, cD galaxies: individual (NGC 4373) - galaxies: stellar content
\end{abstract}

\section{INTRODUCTION}

The measurement of surface brightness fluctuations (SBF) in early-type galaxies (Tonry \& Schneider 1988; Jacoby et al. 1992) is an important development in the investigation of the extragalactic distance scale. The rms

\footnotetext{
${ }^{1}$ Based on observations with the NASA/ESA Hubble Space Telescope obtained at the Space Telescope Science Institute, which is operated by the Association of Universities for Research in Astronomy, Inc., under NASA contract NAS 5-26555.

${ }^{2}$ Palomar Observatory, California Institute of Technology, 105-24 Pasadena, CA 91125.

${ }^{3}$ Present address: Harvard-Smithsonian Center for Astrophysics, 60 Garden Street, Mail Stop 20, Cambridge, MA, 02138; mpahre@cfa.harvard.edu.

${ }^{4}$ Hubble Fellow.

${ }^{5}$ Mount Stromlo and Siding Spring Observatories, Institute of Advanced Studies, Australian National University, Private Bag, Weston Creek Post Office, ACT 2611, Australia; jrm@merlin.anu.edu.au.

${ }^{6}$ Observatories of the Carnegie Institution of Washington, 813 Santa Barbara Street, Pasadena, CA 91101; dressler@ociw.edu.

${ }^{7}$ Lowell Observatory, Mars Hill Road, Flagstaff, AZ 86001.

${ }^{8}$ Department of Astronomy, University of Wisconsin at Madison, 475 North Charter Street, Madison, WI 53706.

9 Department of Atmospheric and Oceanic Sciences, University of Michigan, 2455 Hayward, Ann Arbor, MI 48109.

${ }^{10}$ Space Telescope Science Institute, 3700 San Martin Drive, Baltimore, MD 21218.

${ }^{11}$ Department of Astronomy, Johns Hopkins University, 3400 North Charles Street, Baltimore, MD 21218.

12 Jet Propulsion Laboratory, 4800 Oak Grove Drive, Mail Stop 241105, Pasadena, CA 91109.

13 Jet Propulsion Laboratory, 4800 Oak Grove Drive, Mail Stop 183900, Pasadena, CA 91109.

${ }_{14}$ Department of Physics and Astronomy, Arizona State University, Tyler Mall, Tempe, AZ 85287.

${ }_{15}$ Division of Geological and Planetary Sciences, California Institute of Technology, Pasadena, CA 91125.
}

scatter in galaxy distance measurements is $\sim 0.18 \mathrm{mag}$, according to a recent large survey (Tonry et al. 1997), demonstrating that this technique is an accurate secondary distance estimator. A variety of investigations have followed in the optical (Tonry, Ajhar, \& Luppino 1989, 1990; Tonry \& Schechter 1990; Tonry 1991; Simard \& Pritchet 1994; Sodemann \& Thomsen 1995; Tonry et al. 1997) and nearinfrared (Luppino \& Tonry 1993; Pahre \& Mould 1994; Jensen, Luppino, \& Tonry 1996) and using Hubble Space Telescope (HST)/WFPC2 (Ajhar et al. 1997; Lauer et al. 1997).

The velocity field in the Local supercluster has been mapped for the first time with a significant signal-to-noise ratio $(\mathrm{S} / \mathrm{N})$ by J. L. Tonry ${ }^{16}$ using ground-based SBF. Similar ground-based optical observations at distances greater than $v=3000$ or $4000 \mathrm{~km} \mathrm{~s}^{-1}$, however, are limited by the ability to identify the globular cluster system (see Tonry et al. 1990). This is a result of the residual variance contributed by unidentified globular clusters swamping the SBF signal. For this reason, observations at larger distances have utilized the inversion of the method, i.e., removing the stellar SBF signal (due to the galaxy light) in order to study the globular cluster systems from the remaining SBF signal (e.g., Blakeslee \& Tonry 1995; Blakeslee 1997). In order to measure distances of galaxies at $v>4000 \mathrm{~km} \mathrm{~s}^{-1}$, using the SBF method requires either deep exposures on a very large telescope or substantially improved resolution (or both) in order to characterize the globular cluster system accurately for each galaxy.

Exploring the local velocity field outside the Local supercluster will benefit significantly from the improved angular

\footnotetext{
${ }^{16}$ From J. L. Tonry, 1995, Proceedings of the Heron Island Workshop on Peculiar Velocities in the Universe, http://qso.lanl.gov/ heron/.
} 
TABLE 1

OBSERVED AND DERIVED QUANTITIES FOR THE GALAXIES

\begin{tabular}{|c|c|c|c|c|c|c|c|c|c|c|}
\hline $\begin{array}{l}\text { Galaxy } \\
\text { (1) }\end{array}$ & $\begin{array}{c}v_{g r}^{\mathrm{CMB}} \\
\left(\mathrm{km} \mathrm{s}^{-1}\right) \\
(2)\end{array}$ & $\begin{array}{c}F_{100} \\
\left(\mathrm{MJy} \mathrm{sr}^{-1}\right) \\
(3)\end{array}$ & $\begin{array}{c}A_{B} \\
(\mathrm{mag}) \\
(4)\end{array}$ & $\begin{array}{c}E\left(V-I_{C}\right) \\
(\mathrm{mag}) \\
(5)\end{array}$ & $\begin{array}{c}A_{\mathrm{F} 814 \mathrm{~W}} \\
(\mathrm{mag}) \\
(6)\end{array}$ & $\begin{array}{c}\left(V-I_{C}\right)_{\text {obs }} \\
(\mathrm{mag}) \\
(7)\end{array}$ & $\begin{array}{c}\left(V-I_{C}\right)_{0} \\
(\mathrm{mag}) \\
(8)\end{array}$ & $\begin{array}{l}t \\
(s) \\
(9)\end{array}$ & $\begin{array}{c}I_{\mathrm{F} 814 \mathrm{~W}, 0} \\
(\mathrm{mag}) \\
(10)\end{array}$ & $\begin{array}{c}(m-M)_{0} \\
(\mathrm{mag}) \\
(11)\end{array}$ \\
\hline NGC $3379 . . . .$. & 1142 & 2.3 & 0.164 & 0.049 & 0.079 & 1.261 & 1.212 & 1340 & 28.81 & 30.14 \\
\hline NGC $4406 \ldots \ldots$. & 1493 & 2.4 & 0.171 & 0.051 & 0.082 & 1.257 & 1.206 & 1500 & 29.83 & 31.20 \\
\hline NGC $4373 . . . .$. & 3625 & 6.7 & 0.479 & 0.144 & 0.229 & 1.302 & 1.158 & 6500 & 31.31 & 32.99 \\
\hline
\end{tabular}

Note.-Col. (2) is from Faber et al. (1989). The $\left(V-I_{C}\right)$ colors for NGC 3379 and NGC 4406 are taken from Ajhar et al. (1997) and corrected to match the extinction in col. (5), whereas $\left(V-I_{C}\right)$ for NGC 4373 is taken from Dressler et al. (1999). Measurements of $I_{\mathrm{F} 814 \mathrm{w}}$ in col. (11) are taken from Table 2 along with the correction for Galactic extinction from col. (6).

resolution available from $H S T$. To understand the opportunity provided by Wide-Field/Planetary Camera-2 (WFPC2) for this purpose, the Investigation Definition Team (IDT) has carried out some pilot observations in NGC 4373, an elliptical galaxy in the Hydra-Centaurus supercluster. This galaxy was selected as a good candidate for SBF observations because of its distance, which at $c z \sim 3625 \mathrm{~km} \mathrm{~s}^{-1}$ puts it at or beyond the limit of groundbased optical SBF measurements. Its location in the Centaurus region on the front-side of the "Great Attractor" (e.g., in the models of Lynden-Bell et al. 1988) makes this galaxy additionally interesting.

When we began this study, it was unknown if the Planetary Camera of WFPC2 could measure SBF accurately,

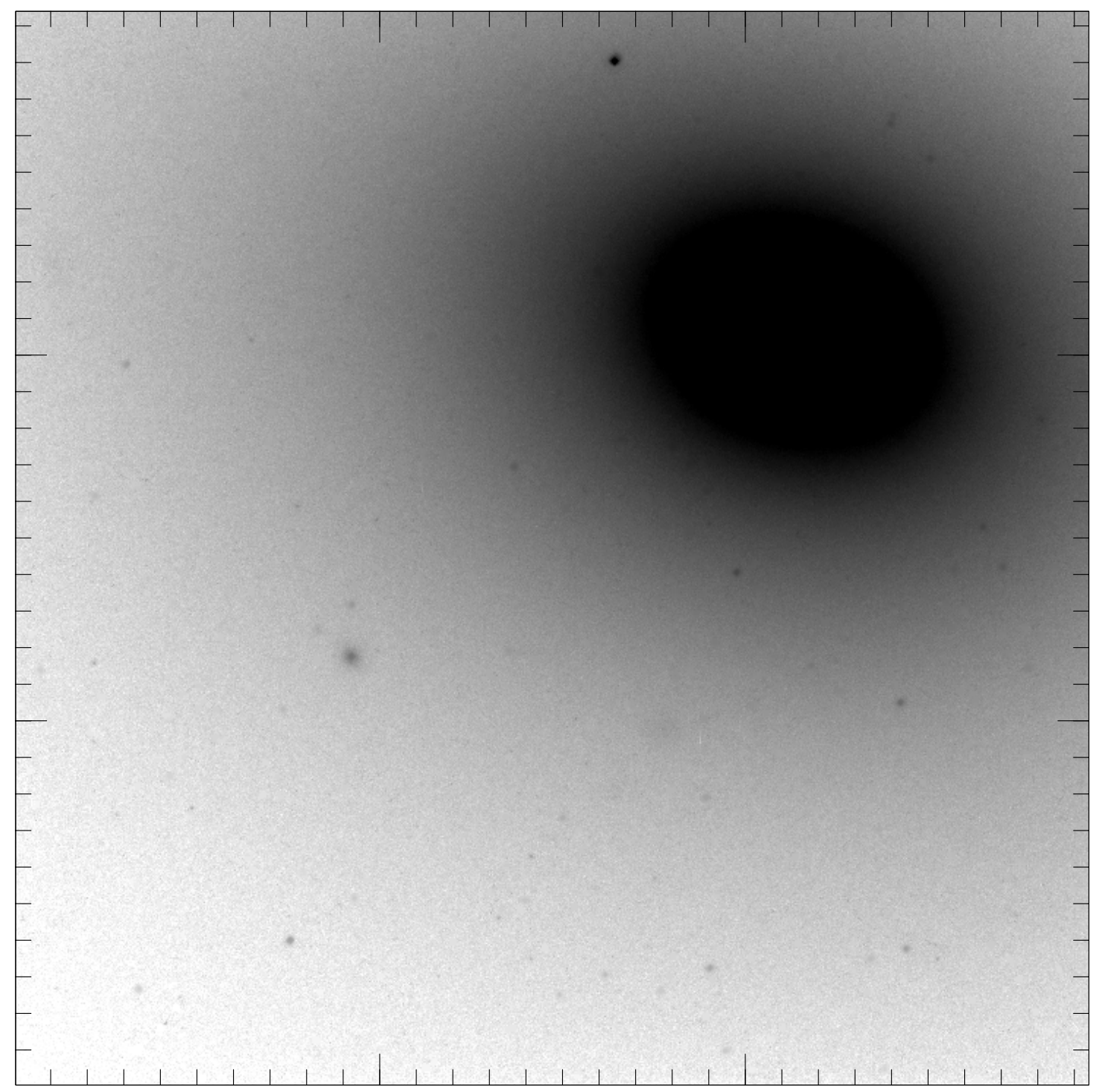

FIG. 1.-Planetary Camera gray-scale image of NGC 4373 displayed with a logarithmic stretch. The small tick marks on both axes are spaced by $1^{\prime \prime}$, while the large tick marks are spaced by $10^{\prime \prime}$. 
because the pixel size only marginally samples the pointspread function (PSF) in the relevant filter F814W (roughly corresponding to the $I_{C}$-band). In this paper, we will demonstrate the feasibility of measuring SBF using HST by analyzing images of two relatively nearby elliptical galaxies-NGC 3379 in the Leo group and NGC 4406 in the Virgo cluster - and comparing the results with groundbased SBF measurements. Then the SBF analysis will be applied to NGC 4373 to measure its distance, thereby showing the power of $H S T$ to extend this secondary distance indicator to larger distances, a conclusion reached independently and contemporaneously by Lauer et al. (1998).

\section{DATA REDUCTIONS}

Images of galaxy NGC 4373 were obtained through the F814W filter using the WFPC2 on the HST as part of a guaranteed time observation (GTO) program. There were five different exposures taken: $2 \times 1900 \mathrm{~s}, 2 \times 1100 \mathrm{~s}$, and $500 \mathrm{~s}$. Additional images of galaxies NGC 3379 in the Leo group and NGC 4406 in the Virgo cluster, also taken through the F814W filter, were obtained from the HST archive. All of the data were obtained using the inverse gain of $7 e^{-} \mathrm{DN}^{-1}$ electronics. The NGC 3379 and NGC 4406 exposures utilized the pipeline processing from the HST archive, while the NGC 4373 exposures were separately processed in a similar way, including ADC correction, bias subtraction, dark subtraction (using super and delta dark frames), and flattening. The frames were then shifted by integer pixel values (to prevent correlating the noise in adjacent pixels) and combined using various cosmic-rayrejection algorithms. There were two separate pointings observed for NGC 4373 that were intentionally offset by an integer number of pixels in order that the images could be reregistered without resampling. The chosen algorithm for combining the images was the STSDAS/CRREJ task, although there was very little difference between the result of this and the IRAF/IMCOMBINE task. The FWHM of the PSF in the final images ranged from $\sim 1.7$ pixel for NGC 4373 to $\sim 2.0$ pixel for the other two galaxies.

The zero point used for the subsequent analysis of NGC 4373 was obtained from Holtzman et al. (1995b) for transforming the $\mathrm{F} 814 \mathrm{~W}$ onto $I_{\mathrm{F} 814 \mathrm{~W}}$ using the color $\left(V-I_{C}\right)=$ 1.23 (to be consistent with Ajhar et al. 1997), producing a calibration of

$$
I_{\mathrm{F} 814 \mathrm{~W}}=21.546-2.5 \log \mathrm{DN}+2.5 \log (t / s),
$$

where $t$ is the integration time. Note that we have not included an additional $0.1 \mathrm{mag}$ into this calibration (as was done by Ajhar et al. 1997), since we will normalize the PSF flux to an aperture of $r=0$ ".5 (see $\S 3.5$ below), although this will not affect the final result. Despite the second-order color term that enters into this equation, the resultant zero point only varies by $<0.01 \mathrm{mag}$ across the relevant range of observed color $1<\left(V-I_{C}\right)<1.4 \mathrm{mag}$.

The $100 \mu \mathrm{m}$ cirrus emission in the region toward and around the three galaxies was obtained for $3 \times 3$ grids centered on the galaxies with $\sim 8^{\prime}$ spacing using the $I R A S$ m.eps. Obvious point sources were visually excluded. The conversion to extinction is then

$$
A_{B}=\frac{F_{100}}{14 \pm 2 \mathrm{MJy} \mathrm{sr}^{-1}} \mathrm{mag}
$$

(Laureijs, Helou, \& Clark 1994). The relative extinction was assumed to be $A_{\mathrm{F} 814 \mathrm{~W}}=0.479 A_{B}$ (Holtzman et al. 1995b) and $E_{V-I}=0.301 A_{B}$ (Cardelli, Blayton, \& Mathis 1989). The $A_{B}$ extinction derived for NGC 4373 is 0.11 mag larger than was assumed by Faber et al. (1989).

The exposure times, extinction estimates, and the observed and dereddened colors for the three galaxies are listed in Table 1. A gray-scale representation of the Planetary Camera image of NGC 4373 is shown in Figure 1.

\section{SBF ANALYSIS}

\subsection{Fitting a Model to the Galaxies}

A first estimate of the mean galaxy profile was constructed using the ELLIPSE task in the ISOPHOTE package of STSDAS. For NGC 4373 only, this profile was rebinned at large semimajor axis $(a>30$ pixels) in order to improve the $\mathrm{S} / \mathrm{N}$, following the procedure of Djorgovski (1985). The profile was fitted to an $a^{1 / 4}$ de Vaucouleurs form at small semimajor axis values $\left(1^{\prime \prime}<a<6^{\prime \prime}\right)$ where the estimated ratio of sky to galaxy is $<4 \%$; this fit was extrapolated to large semimajor axes values to determine the deviation of the profile due to the sky brightness. The surface brightness profile and the residuals from the $a^{1 / 4}$ model fit are shown in Figure 2.

The median predicted offset due to the sky brightness was then estimated for the extrapolated region to be $153 \pm 10$

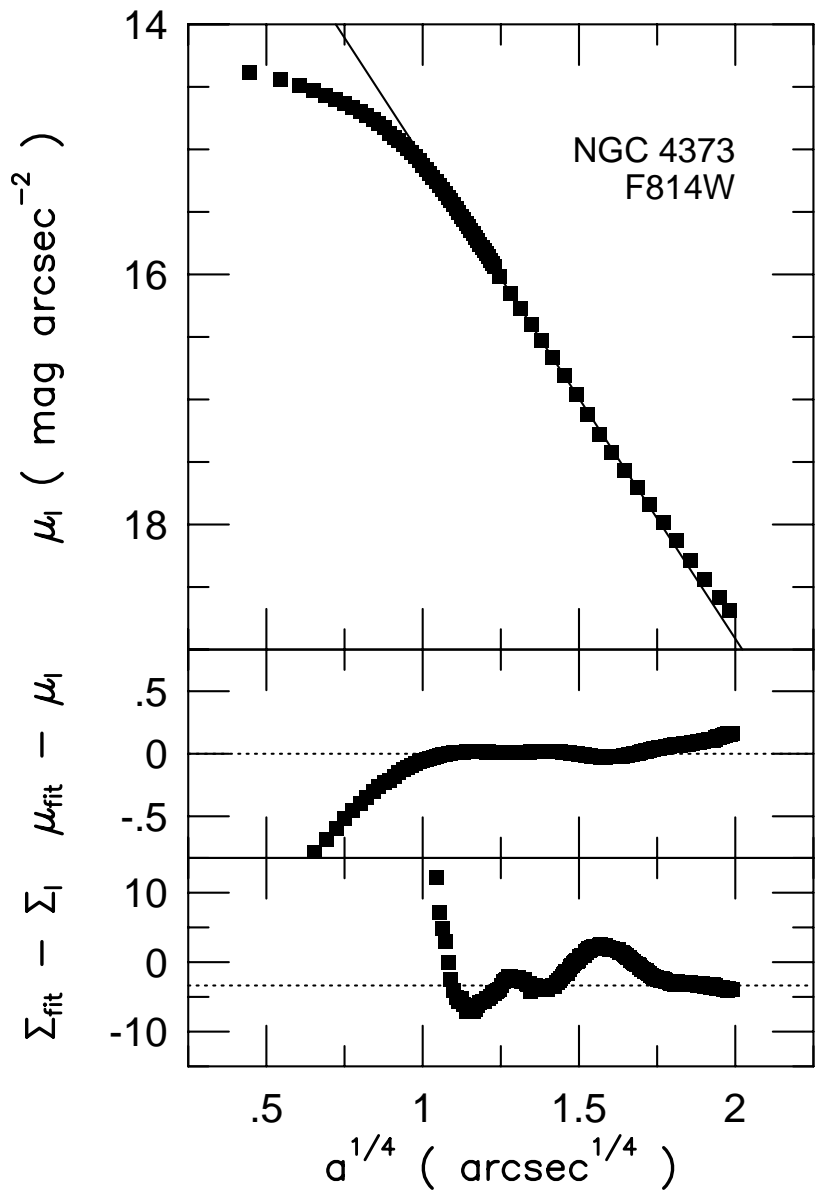

FIG. 2.- Surface brightness profile with fit to a de Vaucouleurs $a^{1 / 4}$ form for $1^{\prime \prime}<a<6^{\prime \prime}$. Residuals in the surface brightness from this fit are given in mag $\operatorname{arcsec}^{-2}$ (middle) and DN pixel ${ }^{-1}(1000 \mathrm{~s})^{-1}$ (bottom). The median sky value of $3.36 \mathrm{DN}$ pixel $^{-1}(1000 \mathrm{~s})^{-1}$ (equivalent to $153 e^{-}$in $6500 \mathrm{~s})$ is shown with a dotted line in the bottom panel. 
$e^{-}$for the $6500 \mathrm{~s}$ exposure, where the uncertainty is the quartile-estimated scatter of the residuals under the assumption that they are normally distributed. Since it is apparent from Figure 2 that the sky value is clearly a systematic error instead of a random one, comparisons of that sky value with other estimates can offer a more reasonable estimate of the sky subtraction uncertainty. We examined the WF3 stacked image and estimated a sky value of $98 e^{-}$ in the PC1 image. An estimate of the sky brightness from PC1 images taken with F814W on blank portions of sky (i.e., several high-redshift clusters and deep field galaxy surveys) puts a typical sky brightness at $135-180 e^{-}$in 6500 s. Finally, we used a separate data reduction package (see $\S$ 3.5 below) to measure the sky value using an extrapolated de Vaucouleurs profile, resulting in a sky value of $198 e^{-}$. The first estimate of $153 e^{-}$was therefore judged to be reasonable, and this was subtracted from the image itself. A new estimate of the mean galaxy profile was then constructed as before. The other two galaxies, NGC 3379 and NGC 4406, had a ratio of sky to galaxy of $3 \%-5 \%$ at $a=300$ pixels (13".7). Accurate sky subtraction is relatively unimportant for these galaxies, because the uncertainty of the sky subtraction will enter the fluctuations image as the square root of the fractional sky contribution to the signal.

A model image was then constructed from this profile using the first through fourth Fourier coefficients of the fit. The model was subtracted from the image to construct the residual image, with semimajor axes between $150<a<300$ pixels used; the inner limit was chosen to remove nuclear areas that are poorly fitted by the elliptical model, while the outer limit was required by the limit of the profile-fitting procedure (the nucleus for NGC 4373 is offcenter on the PC1 FOV). There is some large-scale structure remaining in the image, as is typical, since elliptical models are not perfect representations of elliptical galaxy light distributions.

Point-sources were then masked-out in the residual image, which was smoothed on a scale of ten times the PSF (Tonry \& Schneider 1988); this smoothed residual image was then subtracted from the residual image to remove the large-scale structures. As a result of this step in the procedure, the low wavenumbers in the power spectrum are compromised and will have to be excluded from the fit to the fluctuations variance. Since the PSF size is $\sim 2$ pixel FWHM, corresponding to a Gaussian width of $\sim 0.85$ pixels, this results in a smoothing kernel of $\sim 10$ pixels for a 512 pixel Fourier transform (FT). The variance of this smoothing kernel in the power spectra will fall off rapidly becoming negligible at $>3 \sigma$, or $k>30$ in the FT, and so wavenumbers below this value will be excluded from the fit to the residual variance (see $\S 3.3$ ).

\subsection{Identifying Point Sources}

The residual image was then searched for sources using DAOPHOT (Stetson 1987). The region within $a<150$ pixels presents difficulty for the detection scheme in DAOPHOT, because there is still some residual large-scale structure remaining after the galaxy subtraction. Hence only the region at $150<a<300$ pixels was searched for sources and will be used in the fluctuations analysis. The threshold was set for $5 \sigma$, which corresponds roughly to $I_{\text {cut }}=25.0 \mathrm{mag}$ for NGC 4373 and $I=24.2 \mathrm{mag}$ in the other two galaxies. Experiments were performed with different values of $I_{\text {cut }}$, which demonstrate that the final distance measurement for NGC 4373 is altered by $<0.04$ mag in all cases and $<0.02$ mag in most cases. A total of 52, 14, and 8 sources were identified within this annulus for NGC 4373, NGC 4406, and NGC 3379, respectively. The PSF was constructed using the brightest sources detected in this annulus and was determined to be fitted best by an analytical expression of the "Lorentz" form. The PSF fit for NGC 4373 is dominated by the brightest source near the top of the frame, which is presumably a foreground galactic star.

The completeness of the detection algorithm was estimated by adding sources back into the residual image using ADDSTAR and then determining what fraction at a given magnitude are recovered. This procedure was done in detail for NGC 4373, and its results were rescaled for use on the other two galaxies. As will be seen below, the residual variance due to unidentified sources in these residual images is small compared with the variance of the fluctuations in all three galaxies. These simulations suggested that $97 \%$ of sources are recovered in the NGC 4373 image at $I=23.75 \mathrm{mag}, 86 \%$ at $I=24.25 \mathrm{mag}$, and $38 \%$ at $I=24.75$ mag. The recovery rate is worse than is expected from the random noise because of the sky brightness, the added noise of the galaxy signal, and weak, large-scale structures in the residual image.

A globular cluster luminosity function (GCLF) was fitted to the detected sources simultaneously with the expected number counts of background galaxies. The latter was estimated from WFPC2 number counts:

$$
\frac{d N}{d m_{\mathrm{I}}}=24010^{0.37(I-17)} \mathrm{deg}^{-2} \mathrm{mag}^{-1}
$$

(Cowie, Hu, \& Songaila 1995), which fits both the groundbased and WFPC2 number counts up to $I<26 \mathrm{mag}$. The GCLF was assumed to have a Gaussian shape (i.e., $a_{0} \exp$ $\left\{-\left[\left(I-a_{1}\right) / 2^{1 / 2} a_{2}\right]^{2}\right\}$; Harris 1988) with the center magnitude fixed. The assumed parameters were $M_{V}=-7.1 \mathrm{mag}$, $a_{2}=\sigma=1.4 \mathrm{mag}$, and $(V-I)=1.1$ for the GCLF.

A relative distance of 2.3 greater than the Virgo cluster was assumed for NGC 4373, while distance moduli of 31.15 and 30.30 were assumed for the Virgo cluster and Leo group, respectively. This value for the center magnitude of the GCLF is usually determined by iteration once a first measurement of the distance to the galaxy is complete. As the residual variance due to unidentified sources is quite small (see below), there is little need to iterate to improve these first guesses for the distances. The fit for the GCLF was done using nonlinear iteration of the LevenbergMarquardt Method, as implemented by Press et al. (1986). Convergence was reached rapidly, producing the fitted value for the amplitude of the Gaussian. The cutoff for the detected sources was taken as $I=25.0$ mag for NGC 4373 and $I=24.0 \mathrm{mag}$ for the other two galaxies. Uncertainties were included in the fit according to Poisson number statistics, with detected sources ranging between 1 and 12 per $0.5 \mathrm{mag}$ bin. The detected point sources from DAOPHOT and the fitted GCLF are shown in Figure 3 for NGC 4373.

\subsection{Calculating the Fluctuation Signal}

The next step in the SBF analysis is to create a mask for the image. All detected sources are masked up to a chosen limiting magnitude $I_{\text {cut }}=25.0 \mathrm{mag}$. Various annuli are also chosen, which here we shall take only $150<a<200$ pixels, $200<a<250$ pixels, and $250<a<300$ pixels, where the 


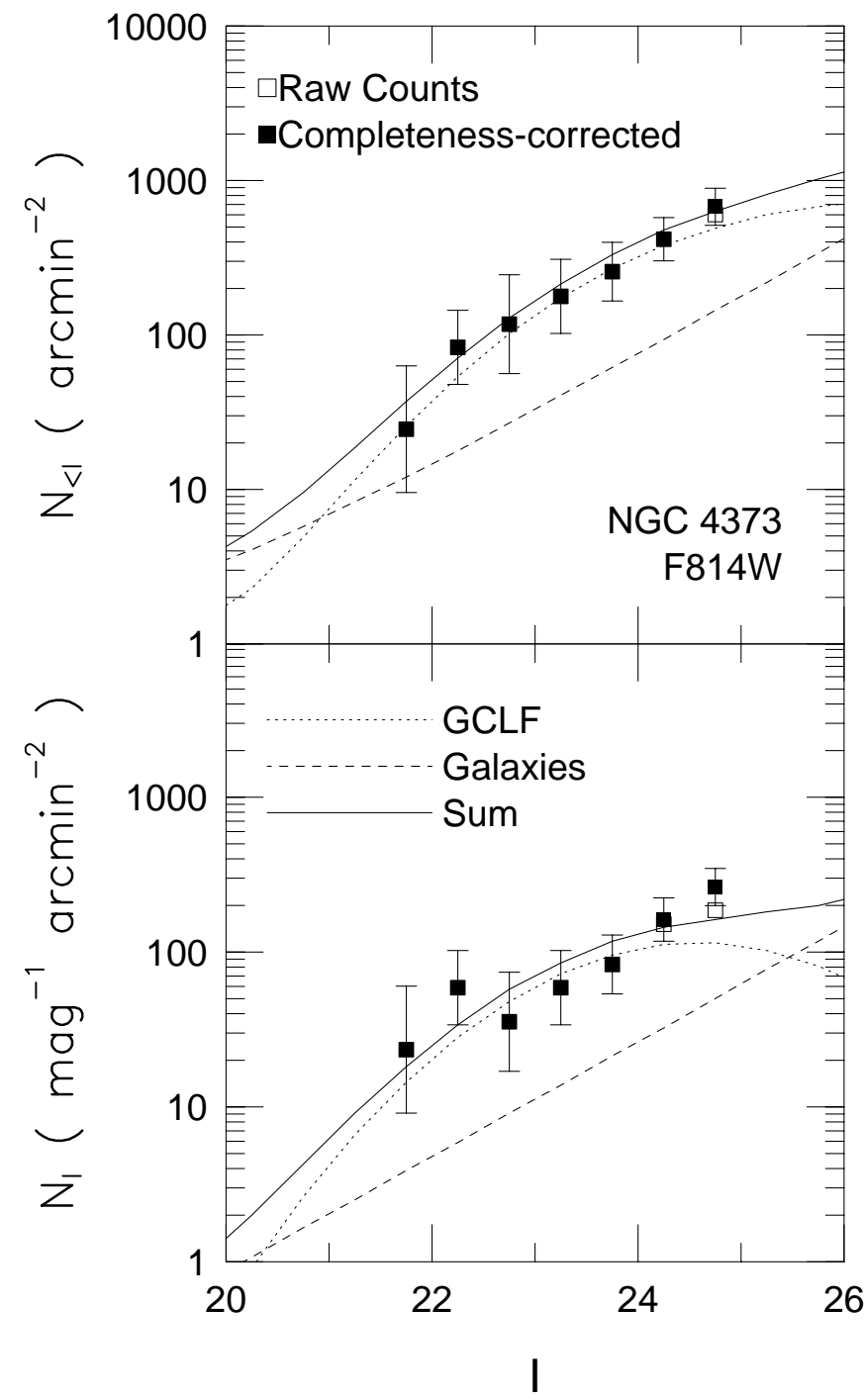

FIG. 3.-Luminosity function for the DAOPHOT detected objects within the annulus having $150<a<300$ pixels. Detections are shown by open symbols, and filled symbols show the detections after correction for completeness. The lines show the fitted model for the GCLF, galaxy number counts, and their sum.

semimajor axis $a$ is chosen rather than radius in order to construct an annulus of uniform noise. The residual image, with the large-scale structures removed as described above, is then divided by the square root of the mean galaxy image to normalize the fluctuations in each pixel. This is now the masked fluctuations image.

A bright version of the PSF was constructed from DAOPHOT as described above; it was placed into a copy of each of the masked fluctuations images, in order that the window function used for the fluctuations and the PSF be identical. We compared the results of the above method to an alternate approach (Tonry \& Schneider 1988) of calculating the FT of the window function and introducing this FT separately into the power spectrum of the fluctuations. The differences between these two approaches were found to be negligible.

We note that it is certainly possible to construct a PSF for WFPC2 images using the "Tiny Tim" package, but we strongly prefer the use of an empirical PSF from the same image as the galaxy, since any subtle telescope jitters or defocusing will be accounted for properly. A region of $512 \times 512$ pixels was then extracted from each fluctuations image and PSF image, and the power spectrum was calculated. Circularly symmetric, azimuthally averaged radial profiles about wavenumber $k=0$ were then extracted from the power spectra of the fluctuations and the PSF.

The azimuthally averaged power spectra for the luminosity fluctuations are weighted by the inverse of the fraction of each image that is nonzero in the mask. The azimuthally averaged power spectra for the PSF are normalized so that each represents exactly $1 e^{-}$within an aperture of $r=11$ pixels $=0$ ". 50 . This is a crucial step, because the calibration of Holtzman et al. (1995b) is normalized for the PSF in a circular aperture of precisely this radius. Thus we can use the zero point from Holtzman et al. directly via equation (1). Each fluctuations power spectrum $E(k)$ is represented by

$$
E(k)=P_{0} \times E_{\mathrm{psf}}(k)+P_{1},
$$

where $P_{0}$ represents the multiplicative factor for the PSF that is the distance-dependent fluctuations magnitude and $P_{1}$ is the flat power spectrum of the Gaussian "white" noise. A linear least-squares fit is performed to the data for wavenumbers $30<k<256$, since a step in the reduction process (noted above) compromises the wavenumbers $k<30$.

\subsection{Removal of Variance Due to Unidentified Sources}

The variance expected from point sources not detected in the residual image must be subtracted individually from each measurement of $P_{0}$. By integrating the variance due to undetected sources both brighter than $I_{\text {cut }}$ (because of incompleteness) and fainter than $I_{\text {cut }}$ using the GCLF measured in $\S 3.2$, the result is the residual variance $P_{\text {res }}$ for each annulus. ${ }^{17}$ Because the correction for the residual variance due to unidentified sources is small compared to the fluctuation signal, we did not attempt to fit the data in any other method that would not require binning the detected sources. Residual variance due to undetected sources was estimated by following the GCLF and extragalactic number counts to large magnitudes, and the result was found to converge very quickly at $I>25 \mathrm{mag}$.

Since there is a limited number of sources detected throughout the entire annulus of $150<a<300$ pixels, it would be very challenging to estimate the GCLF for smaller annuli (i.e., separately for $150<a<200,200<a<250$, and so on). The GCLF is expected to vary with projected radius from the galaxy nucleus, so it is clearly not constant across the entire area. Inspection of the radial dependence of the GCLF in well-studied galaxies (such as Cohen 1988 for NGC 4406) suggests that this effect is virtually negligible

\footnotetext{
${ }^{17}$ While the detection algorithm of DAOPHOT may not be $100 \%$ complete in detecting $5 \sigma$ objects in the residual image (Sodemann \& Thomsen 1995), it is $100 \%$ complete at detecting $5 \sigma$ peaks in the image, and so the latter are used for the correction of the residual variance. Since the WFPC2 data are very deep in their removal of point sources, reaching $I_{\text {cut }}=25.0 \mathrm{mag}$ at the $5 \sigma \mathrm{CL}$, any incompleteness in detecting these peaks is irrelevant to the calculation of the residual variance. Ground-based imaging, on the other hand, will generally not reach these faint flux limits in the detection of point sources, so the effects of incompleteness in detecting these $5 \sigma$ peaks on the residual variance become relevant at significant cosmological distances (and faint flux limits) and must be estimated carefully.
} 
for the small range in radii we have studied here. The corrected SBF power is then simply $P_{0}^{\text {corr }}=P_{0}-P_{\text {res }}$.

\subsection{Consistency Checks}

A number of consistency checks were investigated for the NGC 4373 data. First, the first wavenumber in the fit was varied between $k=30$ and $k=70$, since the lowest wavenumbers contribute the largest effect to the $\chi^{2}$ minimization routine. Second, $P_{1}$ was fitted for the largest wavenumbers $(225<k<256)$ and fixed to this value; the implied measurement of $P_{0}$ was then calculated for each individual data point in the range $30<k<70$. Both of these tests suggest that there are no significant individual outliers in the power spectra data, nor are there systematic errors arising from a particular range of wavenumbers. Furthermore, these tests demonstrate that we have identified the appropriate minimum wavenumber for the fits, because lower wavenumbers (at $k<30)$ show clear systematic differences from the remainder of the power spectra.

The effects of the estimate of the sky value were investigated for NGC 4373, the galaxy for which the uncertainty will be greatest, by using the different estimates described in $\S 3.1$. The extreme estimates of the sky value result in a change of $\pm 0.02 \mathrm{mag}$ in the distance estimate, in such a way that the lower sky value produces the further distance estimate.

The entire fluctuations analysis was repeated using an independent software package, written by J. Tonry and provided to A. Dressler. This package can be considered the "standard" for the field (see Tonry et al. 1997 and references therein) and was used for the ground-based $I_{C}$-band distance measurements of galaxies in the Hydra-Centaurus supercluster by Dressler (1993). There are a number of significant differences between the Tonry package and the one we have utilized above: (1) The Tonry package finds sources using a modified DOPHOT package (Mateo \& Schechter 1989; Schechter, Mateo, \& Saha 1993) that includes the SBF in the noise model for detecting sources. (2) The completeness at $5 \sigma$ is taken in the Tonry package to be $100 \%$, which was verified in simulations (J. Tonry 1997, private communication); we have explicitly measured the completeness via simulations and account for any incompleteness near $I_{\text {cut }}$ in the variance due to unidentified sources (which follows closely the method of Sodemann \& Thomsen 1995). Both of these points, however, are relatively unimportant, since the correction itself for the residual variance is small even for NGC 4373. (3) The Tonry package uses an analytical model fit for the power spectrum of the PSF instead of the power spectrum itself (i.e., a fully empirical PSF) that we have used above. (4) The image regions used for the Tonry package are somewhat different from those used above. Comparisons were therefore made for the identical subregions of the image, namely, circular annuli of 32:64, 64:128, and 128:256 pixels. (5) The Tonry package in this version normalizes the PSF flux to effectively infinite aperture, while our analysis above normalized the PSF flux to unity within a $r=0.5$ aperture. Using the encircled energy curves for the F814W filter from Holtzman et al. (1995a), the difference is $\sim 9 \%$ in intensity, which we have applied to the Tonry package for the purposes of this comparison. ${ }^{18}$

\footnotetext{
${ }^{18}$ Ajhar et al. (1997) added a $0.1 \mathrm{mag}$ term for this same reason in calibrating their SBF analysis using the Tonry package (i.e., their eq. [2]).
}

Given the subtle but significant differences between these two analyses of the same image, the differences in $P_{0}$ are only $\sim 2 \%$ for NGC 4373 . This is an acceptable systematic error, which is substantially smaller than the overall random uncertainties for the SBF method (Tonry et al. 1997).

\subsection{Converting $I_{\mathrm{F} 814 \mathrm{w}}$ into Distances}

Ajhar et al. (1997) report a calibration of $\bar{M}_{I_{\mathrm{F} 814 \mathrm{~W}}}$ based on detailed comparisons with the large, ground-based study of $I_{C}$-band SBF of Tonry et al. (1997). As was shown by Tonry (1991), there exists a dependence of $\bar{M}_{I_{C}}$ on intrinsic color $(V-I)_{0}$, which was modeled using simple stellar populations by Worthey (1994). The subsequent primary calibration of the SBF distance scale was determined by Ajhar et al. (1997) to be

$$
\bar{M}_{I_{\mathrm{F} 814 \mathrm{~W}}}=-1.73( \pm 0.07)+6.5( \pm 0.7)\left[(V-I)_{0}-1.15\right] \text {. }
$$

The reddening estimates listed in Table 1 are used to convert $(V-I)_{\text {obs }}$ into $(V-I)_{0}$ for the three galaxies.

There is an additional cosmological correction ought to be applied. The $k$-correction for fluctuations observed in the ground-based $I_{C}$-band filter is $7.0 \times z$ (Tonry et al. 1997), which acts to brighten the observed magnitudes. We note that SBF magnitudes act as luminosities, not surface brightnesses, hence there is no need for additional SB dimming cosmological corrections. Furthermore, the broadband color $(V-I)_{\text {obs }}$ needs to be made bluer by $0.9 z$. Since these two effects nearly cancel each other in equation (5), the possible systematic error on the distance modulus for NGC 4373 implied by not making these corrections is only 0.014 mag, which is comparable to the uncertainties of the $k$ corrections themselves (and their applicability to the F814W filter). For this reason, $k$-corrections were not applied in the photometric calibration.

\section{RESULTS}

\subsection{NGC 3379 in the Leo Group}

The fit for the intermediate annulus $(200<a<250$ pixels) in NGC 3379 is displayed in Figure 4, and the fits for $P_{0}, P_{1}$, and $P_{\text {res }}$ are listed in Table 2 . For the three annuli, the mean value after correction for the residual variance due to unmasked sources is $P_{0}^{\text {corr }}=10.8 \pm 0.5 e^{-}$in the $1340 \mathrm{~s}$ exposure, where the uncertainty reflects the scatter among the three measurements divided by $3^{1 / 2}$. An estimate of the $\mathrm{S} / \mathrm{N}$ of a fluctuations measurement is provided by the ratio $\eta=P_{0} / P_{1}$, which we measure to range between $\eta=3.7$ and 8.6 for these annuli.

While the results listed in Table 2 suggest that there might be evidence for radial gradients for our measurements, we caution against this interpretation without a more thorough investigation of the HST images of this galaxy for all possible radii obtainable with the PC array. We have merely chosen to analyze NGC 3379 for only those three annuli identical to those used for NGC 4373 below, as a comparison sample. The scatter among the three individual measurements listed in Table 2 is $0.11 \mathrm{mag}$, which is comparable to the scatter in $I_{C}$ among the entire SBF survey of Tonry et al. (1997) of $\sim 0.1 \mathrm{mag}$. Thus, if a gradient exists, it would only be significant at the $2 \sigma(\mathrm{CL})$. An opposite gradient in $I_{C}$ was detected by Sodemann \& 


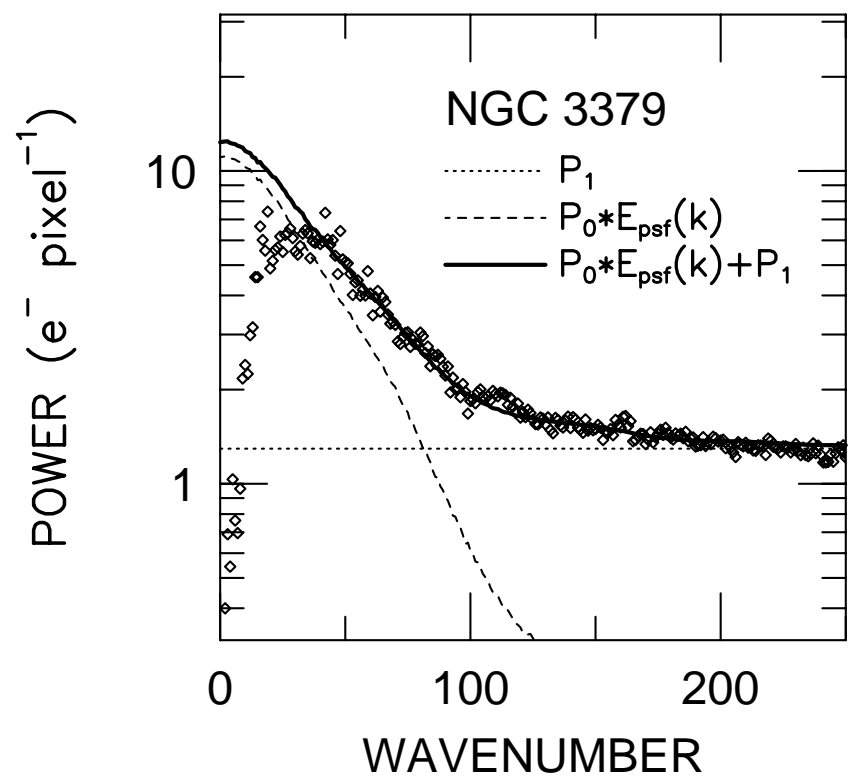

FIG. 4.- Power spectrum of the fluctuations signal in the $200<a<250$ pixels annulus for NGC 3379. The solid line is the fit to the data points using the sum of two terms: a constant $P_{1}$ and a constant $P_{0}$ multiplied by the power spectrum $E_{\text {epsf }}(k)$ of the PSF. The data points at low wavenumber $k<30$ were compromised because of a step in the data reduction method (see $\S 2$ ), and so only the data for $30<k<256$ were used for the fit.

Thomsen (1995) for NGC 3379, although for annuli at much larger radii (13".5-48") than we have studied here. An extrapolation of their results implies that we should detect a brightening in $I_{C}$ of only 0.06 mag between the innermost and outermost annuli in the PC image. A color gradient in $(B-J)$ but not $(J-K)$ was detected by Peletier, Valentijn, \& Jameson (1990), so it is unclear if a gradient in $I_{C}$ should be expected on the basis of color gradients in NGC 3379. Errors in sky subtraction are not nearly large enough to cause the observed effect (see $\S 3.1$ ). Finally, we note that the outermost annulus had a disproportionate number of identified points sources compared to the other annuli, which could cause a subtle measurement bias. More detailed study is required if a fluctuations gradient is to be inferred from the data.

The mean measurement for the fluctuations magnitude for NGC 3379 is $I_{\mathrm{F} 814 \mathrm{~W}}=28.81 \pm 0.11 \mathrm{mag}$ (after correct- ing for Galactic extinction), which results in a distance modulus of $(m-M)_{0}=30.14 \pm 0.15$ mag through application of equation (5). The uncertainty estimate in $I_{\mathrm{F} 814 \mathrm{~W}}$ only represents three uncertainties: the scatter between the measurements for the different regions of the image $(0.11 \mathrm{mag})$, the stability of the WFPC2 zero point for F814W of 0.018 mag (Ajhar et al. 1997), and the $14 \%$ uncertainty in the conversion between IRAS $100 \mu \mathrm{m}$ flux and $A_{B}$ extinction. The uncertainty estimate for $(m-M)_{0}$ includes the uncertainty of $0.01 \mathrm{mag}$ for $\left(V-I_{C}\right)_{\text {obs }}$, the uncertainty of 0.055 mag for the fit between $\left(V-I_{C}\right)_{0}$ and $\bar{M}_{I_{\mathrm{F} 814 \mathrm{~W}}}$, and $0.05 \mathrm{mag}$ to allow for cosmic scatter (Tonry et al. 1997). Our measurement of $(m-M)_{0}$ agrees with the value of $30.08 \pm 0.08$ from the Tonry et al. (1997) ground-based SBF survey, as quoted by Ajhar et al. (1997). It is somewhat nearer, however, than the value derived independently from the WFPC2 data by Ajhar et al. (significant at the $<2 \sigma$ confidence level [CL] after correction for the different assumptions of Galactic extinction).

\subsection{NGC 4406 in the Virgo Cluster}

The fit for the intermediate annulus $(200<a<250$ pixels) in NGC 4406 is displayed in Figure 5, and the fits for $P_{0}, P_{1}$, and $P_{\text {res }}$ are listed in Table 2 . The scatter among the different annuli is remarkably small, with no evidence for a radial dependence for $P_{0}$. Because this exposure time was similar to that of NGC 3379 but this galaxy is in the more distant Virgo cluster, the $\mathrm{S} / \mathrm{N}$ is poorer, ranging between $\eta=1.1$ and 3.9. Nonetheless, the small scatter among the annuli suggests that there are no systematic biases entering into these data as a function of $\eta$.

The mean measurement for the fluctuations magnitude for NGC 4406 is $I_{\mathrm{F} 814 \mathrm{~W}}=29.83 \pm 0.03 \mathrm{mag}$ (corrected for Galactic extinction), which results in a distance modulus of $(m-M)_{0}=31.20 \pm 0.10$. The uncertainties were estimated in the same manner as for NGC 3379 in $\S 4.1$ above. This distance modulus agrees with the value $31.19 \pm 0.07 \mathrm{mag}$ with the Tonry et al. (1997) ground-based SBF measurement as quoted by Ajhar et al. (1997).

\subsection{NGC 4373 in the Hydra-Centaurus Supercluster}

The fits to each of the three annuli are displayed in Figure 6 , and the results are listed in Table 2. The results are from the three annuli that are mutually consistent at the $0.02 \mathrm{mag}$ level. This exposure was longer $(6500 \mathrm{~s})$ than either of the

TABLE 2

FluCtUATIONS ANALysis

\begin{tabular}{cccccccc}
\hline \hline Galaxy & $\begin{array}{c}\text { Annulus } \\
\text { (pixels) }\end{array}$ & $\begin{array}{c}P_{0} \\
\left(e^{-} \text {pixel }^{-1}\right)\end{array}$ & $\begin{array}{c}P_{1} \\
\left(e^{-} \text {pixel }^{-1}\right)\end{array}$ & $\begin{array}{c}P_{\text {res }} \\
\left(e^{-} \text {pixel }^{-1}\right)\end{array}$ & $\begin{array}{c}P_{0}^{\text {corr }} \\
\left(e^{-} \text {pixel }^{-1}\right)\end{array}$ & $\begin{array}{c}I_{\mathrm{F} 814 \mathrm{~W}} \\
(\mathrm{mag})\end{array}$ & $\begin{array}{c}\sigma_{I_{\mathrm{F} 814 \mathrm{w}}} \\
(\mathrm{mag})\end{array}$ \\
\hline NGC $3379 \ldots \ldots$ & $150: 200$ & 11.81 & 1.56 & 0.04 & 11.77 & 28.80 & 0.02 \\
& $200: 250$ & 11.16 & 1.30 & 0.05 & 11.11 & 28.86 & 0.04 \\
& $250: 300$ & 9.68 & 2.64 & 0.05 & 9.63 & 29.02 & 0.04 \\
NGC $4406 \ldots \ldots$ & mean & $\ldots$ & $\ldots$ & $\ldots$ & 10.83 & 28.89 & 0.11 \\
& $150: 200$ & 4.68 & 1.22 & 0.04 & 4.64 & 29.93 & 0.05 \\
& $200: 250$ & 4.88 & 1.25 & 0.05 & 4.83 & 29.89 & 0.02 \\
& $250: 300$ & 4.78 & 4.40 & 0.06 & 4.72 & 29.91 & 0.05 \\
NGC $4373 \ldots \ldots$ & mean & $\ldots$ & $\ldots$ & $\ldots$ & 4.73 & 29.91 & 0.02 \\
& $150: 200$ & 4.61 & 0.96 & 0.05 & 4.56 & 31.54 & 0.02 \\
& $200: 250$ & 4.57 & 1.03 & 0.06 & 4.51 & 31.56 & 0.05 \\
& $250: 300$ & 4.75 & 1.31 & 0.08 & 4.67 & 31.52 & 0.04 \\
& mean & $\ldots$ & $\ldots$ & $\ldots$ & 4.58 & 31.54 & 0.02 \\
\hline
\end{tabular}




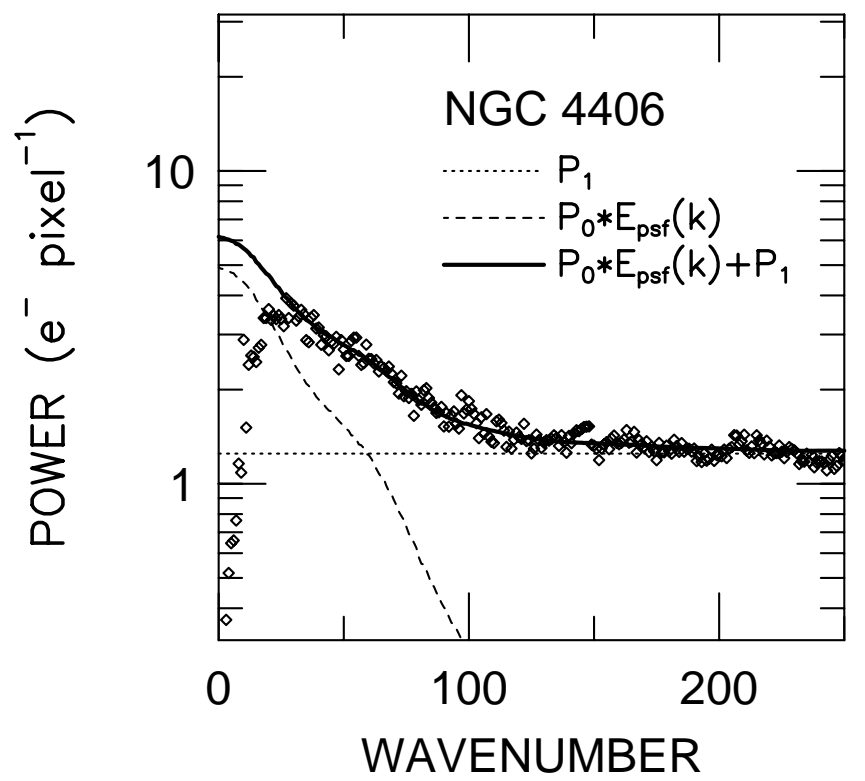

FIG. 5.-Power spectrum of the fluctuations signal in the $200<a<250$ pixel annulus for NGC 4406. This figure is otherwise identical to Fig. 4.

other two galaxies but is further in distance; hence the $\mathrm{S} / \mathrm{N}$ is comparable to the best of NGC 4406, i.e., $\eta=3.6-4.8$.

The mean measurement for the fluctuations magnitude for NGC 4373 is $I_{\mathrm{F} 814 \mathrm{~W}}=31.31 \pm 0.05 \mathrm{mag}$ (corrected for Galactic extinction), which results in a distance modulus of $(m-M)_{0}=32.99 \pm 0.11$. Once again, the uncertainties are estimated as described in $\S 4.1$, with an additional $0.02 \mathrm{mag}$ added because of sky-subtraction uncertainties that are not relevant for the other two galaxies.

Dressler et al. (1999) have made new measurements of the distances to Hydra-Centaurus galaxies using ground-based SBF data obtained under excellent seeing conditions. Preliminary results described by Dressler (1993) for NGC 4373 were obtained with relatively poor seeing (1".03 FWHM) compared to the newer data (0"76 FWHM), so these new measurements supersede the older ones. Using the calibration of Tonry et al. (1997) for these new ground-based data and the same extinction as listed in Table 1, the preliminary distance modulus is 32.93 , in excellent agreement with the value derived from the WFPC 2 observations.

Peculiar velocities should be calculated relative to the baseline expansion rate of $H_{0}=81 \pm 6 \mathrm{~km} \mathrm{~s}^{-1} \mathrm{Mpc}^{-1}$ derived by Tonry et al. (1997) from their SBF database, since their study was used in the present paper for the calibration of $\bar{M}_{I_{\mathrm{F} 814 \mathrm{~W}}} \cdot{ }^{19}$ Taking the velocity of the NGC 4373 group with respect to the cosmic microwave background (CMB) as $3625 \mathrm{~km} \mathrm{~s}^{-1}$ (Faber et al. 1989), the distance modulus of $32.99 \pm 0.11$ suggests that NGC 4373 possesses a peculiar velocity of $v_{\mathrm{pec}}=415 \pm 330 \mathrm{~km} \mathrm{~s}^{-1}$ with respect to the CMB. Alternatively, the ratio of distances between NGC 4373 and NGC 4406 can be compared to the ratio of the CMB relative velocities of the NGC 4373 group and the Virgo cluster (included in Table 1) to calculate a peculiar velocity for NGC 4373. This separate calculation yields a

\footnotetext{
19 The Ajhar et al. (1997) SBF calibration for HST filter F814W was done directly onto the Tonry et al. (1997) system by assuming the distance moduli for each galaxy from the latter study. Hence the two are equivalent for our purpose.
}

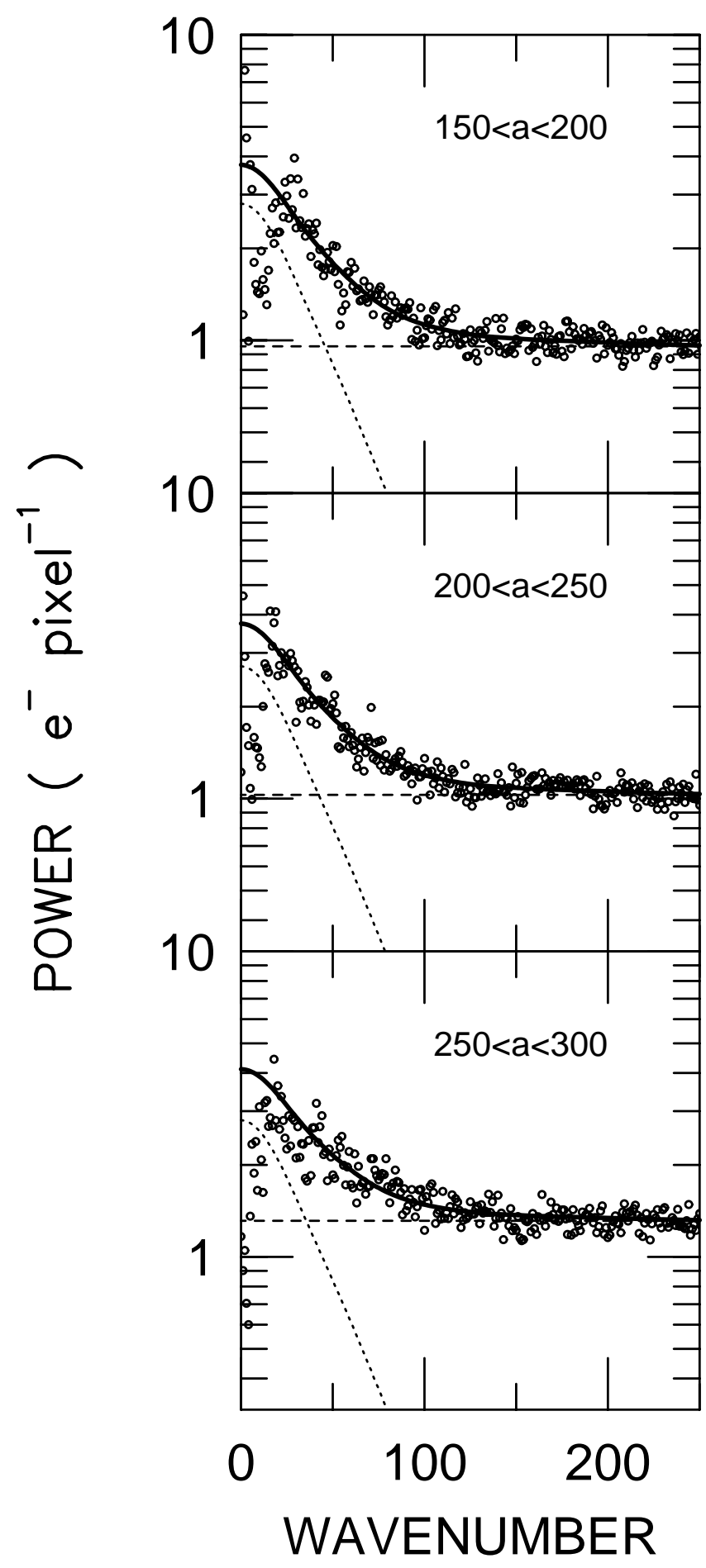

FIG. 6.-Power spectra of the SBF within three different annuli from the F814W image of NGC 4373. The inner and outer semimajor axes of each annulus is indicated in the upper-right portion of each panel. The data are given by open circles, which are fitted as the sum (heavy solid line) of $P_{0}$ times the PSF (dotted lines) and $P_{1}$ (dashed line). The Gaussian noise $\left(P_{1}\right)$ can be seen to increase with semimajor axis, as expected because the fluctuations have been normalized by dividing by the square-root of the mean galaxy flux.

peculiar velocity of $v_{\text {pec }}=220 \pm 270 \mathrm{~km} \mathrm{~s}^{-1}$. The cause of the difference between these two estimates of $v_{\text {pec }}$ is the somewhat high value of the cosmological redshift in the CMB frame for the Virgo cluster $\left(1493 \mathrm{~km} \mathrm{~s}^{-1}\right.$, from Faber et al. 1989); taking the SBF value of this quantity $(1390 \mathrm{~km}$ 
$\left.\mathrm{s}^{-1}\right)^{20}$ results in $v_{\text {pec }}=455 \pm 270 \mathrm{~km} \mathrm{~s}^{-1}$. We adopt the value of $v_{\text {pec }}=415 \pm 330 \mathrm{~km} \mathrm{~s}^{-1}$, because it does not require the assumption of the CMB velocity of the Virgo cluster from the $D_{n}-\sigma$ relation.

\section{DISCUSSION}

\subsection{Feasibility of SBF With HST}

This paper represents the detection of SBF in images taken with the WFPC2 instrument on HST. The images are marginally sampled or slightly undersampled, even using the Planetary Camera. Nonetheless, we have demonstrated that the fluctuations signal is strong with only a modest exposure time at a distance of the NGC 4373 group in the Hydra-Centaurus supercluster.

The measurements of $(m-M)_{0}$ in NGC 3379 and NGC 4406 agree well with ground-based measurements, suggesting that there are no substantial systematic offsets between the marginally sampled HST SBF data and the oversampled ground-based work at uncertainties of less than $10 \%$ in distance. The Planetary Camera of HST is thus a potentially powerful tool for measuring distances using the SBF method.

The removal of point sources (foreground stars and globular clusters) and background galaxies is straightforward. The estimation of the residual variance due to other undetected globular clusters and galaxies is robust, and the correction implied is quite small, typically $2 \%-4 \%$. This is due to the combination of the depth afforded by the highresolution imaging power of $H S T$ and by the small projected areas that have been studied here. Ground-based imaging typically utilizes a much larger FOV for the calculation of $I_{C}$, resulting in a larger number of undetected objects that must be removed from the SBF signal.

Estimating foreground Galactic extinction is an important element of SBF work with HST, because the slope of the dependence of $\bar{M}_{I_{\mathrm{F} 814 \mathrm{~W}}}$ on $\left(V-I_{C}\right)_{0}$ is 6.5 . This causes errors in the distance modulus to be $\Delta(m-M)_{0} \sim 1.5 \Delta A_{B}$, which might seem surprising, since the SBF observations are made at $\lambda=800 \mathrm{~nm}$. The minimal effect of $k$-corrections on $(\bar{m}-\bar{M})$, however, does provide a reduction in additional sources of uncertainty.

\subsection{The Distance to NGC 4373}

Our distance measurement to NGC 4373 ought to be more accurate than ground-based work because of the greater confidence at which we can estimate $P_{\text {res }}$. Experiments with varying values of $I_{\text {cut }}$ and the $\mathrm{S} / \mathrm{N}$ for detecting such sources suggests that the possible variations in $P_{\text {res }}$ are $\leq 4 \%$ of $P_{0}$. Comparison of two different SBF analysis packages suggests that the systematic errors due to methodology are $\$ 2 \%$ in distance, possibly much less if we account for differences in the normalization of the PSF and sky subtraction.

The distance we estimate for NGC 4373 implies a smaller peculiar velocity than has been measured previously. A comparison of the SBF distance estimates with those of the $D_{n}-\sigma$ relation (Faber et al. 1989) and the group velocities (with respect to the CMB) are shown in Figure 7, where the distances and velocities have been calculated relative to the Virgo cluster. The source of the large peculiar velocity of the Hydra-Centaurus supercluster is shown by POTENT m.eps

\footnotetext{
${ }^{20}$ See footnote 16.
}

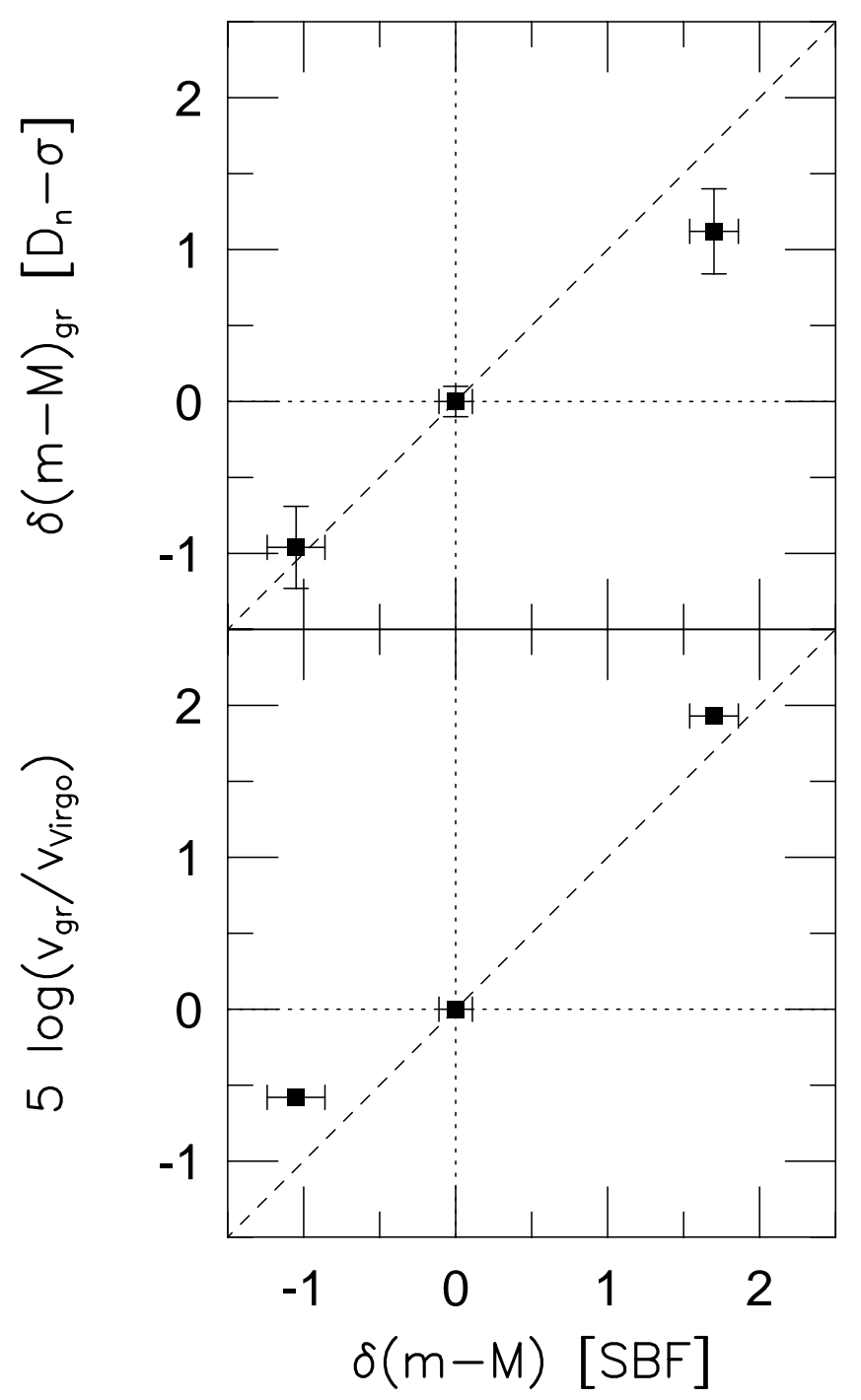

FIG. 7.-Top: Comparison of the SBF distances estimates for the three galaxies with those of each group or cluster using the $D_{n}-\sigma$ relation (Faber et al. 1989). Bottom: Comparison of the SBF distances estimates for the three galaxies with those of each group or cluster expected from the group velocity (from Faber et al. 1989). In both panels, the distances and velocities have been normalized to the Virgo cluster. As seen in the top panel, the SBF distance to NGC 4373 is slightly larger than that from the $D_{n}-\sigma$ relation; part of this difference could be due to NGC 4373 being farther than the other members of its group, as suggested by the ground-based SBF measurements of Dressler (1993).

as a mass concentration at a redshift of $\sim 4500 \mathrm{~km} \mathrm{~s}^{-1} .^{21}$ The model for the velocity flows in this direction based on $D_{n}-\sigma$ observations (Lynden-Bell et al. 1988) predicts a peculiar velocity of $838 \mathrm{~km} \mathrm{~s}^{-1}$ for NGC 4373. Based on TullyFisher distance measurements of clusters in the Hydra-Centaurus region, however, Aaronson et al. (1989) and Mould et al. (1991) measured the median value of the peculiar velocity flow to be 489 and $610 \mathrm{~km} \mathrm{~s}^{-1}$, respectively. Our measured peculiar velocity of $415 \pm 330 \mathrm{~km} \mathrm{~s}^{-1}$ for NGC 4373 is therefore in better agreement with the values derived from the Tully-Fisher method, although the measurement is only in marginal conflict with the model based on the $D_{n}-\sigma$ data. We note that the peculiar velocity to the NGC 4373 group might be somewhat larger if IC

${ }^{21}$ See A. Dekel, 1995, Proceedings of the Heron Island Workshop on Peculiar Velocities in the Universe, http://qso.lanl.gov/ heron/. 
3370 and NGC 4373A are closer than NGC 4373, as implied by the ground-based SBF results of Dressler (1993), although this result ought to be reinvestigated using observations obtained in better seeing conditions.

It is clear from this simple analysis that more SBF measurements of distances, both ground-based and HST, should be attempted to understand better the velocity flows in the Hydra-Centaurus region.
We acknowledge many helpful discussions with J. Blakeslee and G. Worthey, and the insightful comments and careful reading of the manuscript by the referee, J. Tonry. This work was supported by NASA grant NAS7-1260 to the WFPC2 IDT. M. A. P. received partial support from NSF grant AST-9157412 and Hubble Fellowship grant HF01099.01-97A from STScI (which is operated by AURA under NASA contract NAS5-26555).
Aaronson, M., et al. 1989, ApJ, 338, 654

Ajhar, E. A., Lauer, T. R., Tonry, J. L., Blakeslee, J. P., Dressler, A., Holtzman, J. A., \& Postman, M. 1997, AJ, 114, 626

Blakeslee, J. P. 1997, ApJ, 481, L59

Blakeslee, J. P., \& Tonry, J. L. 1995, ApJ, 442, 579

Cardelli, J. A., Clayton, G. C., \& Mathis, J. S. 1989, ApJ, 345, 245

Cohen, J. G. 1988, AJ, 95, 682

Cowie, L. L., Hu, E. M., \& Songaila, A. 1995, AJ, 110, 1576

Djorgovski, S. 1985, Ph.D. thesis, Univ. California at Berkeley

Dressler, A. 1993, in Cosmic Velocity Fields, Proceedings of the 9th IAP Astrophysics Meeting, ed. F. R. Bochet \& M. Lachièze-Rey (Gif-surYvette: Editions Frontieres), 9

Dressler, A., et al. 1999, in preparation

Faber, S. M., Wegner, G., Burstein, D., Davies, R. L., Dressler, A., LyndenBell, D., \& Terlevich, R. J. 1989, ApJS, 69, 763

Harris, W. E. 1988, in The Extragalactic Distance Scale, ed. S. v. d. Bergh \& C. J. Pritchet, 231

Holtzman, J. A., Burrows, C. J., Casertano, S., Hester, J. J., Trauger, J. T., Watson, A. M., \& Worthey, G. 1995b, PASP, 107, 1065

Holtzman, J., et al. 1995a, PASP, 107, 156

Jacoby, G. H., et al. 1992, PASP, 104, 599

Jensen, J. B., Luppino, G. A., \& Tonry, J. L. 1996, ApJ, 468, 519

Lauer, T. R., Tonry, J. L., Postman, M., Ajhar, E. A., \& Holtzman, J. A. 1998, ApJ, 499, 577

Laureijs, R. J., Helou, G., \& Clark, F. O. 1994, in ASP Conf. Ser. 58, Proceedings of The First Symposium on the Infrared Cirrus and Diffuse Interstellar Clouds, ed. R. M. Cutri \& W. B. Latter (San Francisco: ASP), 133

\section{EFERENCES}

Luppino, G. A., \& Tonry, J. L. 1993, ApJ, 410, 81

Lynden-Bell, D., Faber, S. M., Burstein, D., Davies, R. L., Dressler, A., Terlevich, R. J., \& Wegner, G. 1988, ApJ, 326, 19

Mateo, M., \& Schechter, P. L. 1989, in First ESO/ST-ECF Data Analysis Workshop, ed. P. J. Grosbøl, F. Murtagh, \& R. H. Warmels (Garching: ESO), 69

Mould, J. R., et al. 1991, ApJ, 383, 467

Pahre, M. A., \& Mould, J. R. 1994, ApJ, 433, 567

Peletier, R. F., Valentijn, E. A., \& Jameson, R. F. 1990, A\&A, 233, 62

Press, W. H., Flannery, B. P., Teukolsky, S. A., \& Vetterling, W. T. 1986, Numerical Recipes (Cambridge: Cambridge Univ. Press)

Schechter, P. L., Mateo, M., \& Saha, A. 1993, PASP, 105, 1342

Simard, L., \& Pritchet, C. J. 1994, AJ, 107, 503

Sodemann, M., \& Thomsen, B. 1995, AJ, 110, 179

Stetson, P. B. 1987, PASP, 99, 191

Tonry, J. L. 1991, ApJ, 373, L1

Tonry, J. L., Ajhar, E. A., \& Luppino, G. A. 1989, ApJ, 346, L57 . 1990, AJ, 100, 1416

Tonry, J. L., Blakeslee, J. P., Ajhar, E. A., \& Dressler, A. 1997, ApJ, 475, 399

Tonry, J. L., \& Schechter, P. L. 1990, AJ, 100, 1794

Tonry, J., \& Schneider, D. P. 1988, AJ, 96, 807

Worthey, W. 1994, ApJS, 95, 107 